\title{
Dysphagia due to Physiological Constriction after Stroke: A Case Report
}

\author{
Minjoon Bae, M.D., Inhyun Kang, M.D., Yangrok Hur, M.D., Kihun Hwang, M.D. \\ Department of Physical Medicine and Rehabilitation, Dong-Eui Medical Center, Busan, Korea
}

\begin{abstract}
The aortic arch normally causes no symptomatic physiological constriction of the esophagus. Symptoms of dysphagia are generally observed in the presence of specific conditions, such as an aortic aneurysm. In the present case, during a videofluoroscopic swallowing study (VFSS), the authors incidentally found that despite the absence of an aortic lesion, the dysphagia observed in a stroke patient was caused by compression from the aortic arch. The patient complained of discomfort when swallowing, similar to a bolus lodged in the chest, which was consistent with compression by the aortic arch found on the VFSS and chest computed tomography (CT). After undergoing dysphagia therapy, the VFSS after 52 days revealed improved swallowing function; however, the sensation of food lodging in the chest due to compression by the aortic arch persisted. We conclude that although the physiological constriction of the esophagus by the aortic arch is usually asymptomatic, the constriction can be a factor that exacerbates the swallowing function in patients with dysphagia, and should therefore be considered during the differential diagnosis. (JKDS 2021;11:143-146)
\end{abstract}

Keywords: Deglutition disorders, Esophagus, Stroke

\section{INTRODUCTION}

There are several potential structures surrounding the esophagus that could cause physiological constriction; among these, the aortic arch can compress the esophagus from the front. Because food easily passes through these mild constrictions by normal peristalsis, physiological constriction does not generally cause symptoms. Compression by the aorta that is sufficiently severe to cause dysphagia is known as "dysphagia aortica". However, it occurs only when the aorta exhibits lesions such as an aneurysm. The exact incidence of dysphagia aortica has not been determined, although it rarely occurs ${ }^{1}$ and it appears that females are predisposed, and factors including older age, short stature, hypertension, and kyphosis ${ }^{2,3}$ are also suspected. We report a case involving a patient who experienced dysphagic symptoms after stroke that occurred in the physiological constriction area despite the absence of overt lesions in the aorta.

\section{CASE REPORT}

A 76-year-old man diagnosed with stroke visited the hospital for rehabilitation including swallow therapy. He was diagnosed with right frontoparietoo-
Received: March 3 2021, Revised: March 15 2021,

Accepted: March 292021

Corresponding author: Kihun Hwang, Department of Physical Medicine and Rehabilitation, Dong-Eui Medical Center, 62 Yangjeong-ro, Busanjin-gu, Busan 47227, Korea Tel: +82-51-850-8942, Fax: +82-51-867-5162

E-mail: drsheva01@gmail.com
Copyrights (c) The Korean Dysphagia Society, 2021. 
ccipital lobe and basal ganglia infarction 26 days previously, and had a history of hypertension, atrial fibrillation, and hyperthyroidism. On physical examination at admission, his muscle strength, rated according to the medical research council scale, was graded $2 / 2$ in the left upper and lower extremities. He had no facial palsy, no deviations in the tongue or uvula, and his gag reflex was normal.

He never complained of dysphagia before the stroke; however, after the stroke he complained of discomfort resembling a bolus lodged in his chest. The discomfort he experienced occurred only when he swallowed, especially, solid food. He did not complain of dyspnea, and his oxygen saturation was normal (98\%). There was no heartburn, weight loss, anorexia, nausea, or vomiting. A VFSS was performed to evaluate swallowing function on the next day using $5 \mathrm{ml}$ of liquid, yoplait, a thick fluid and solid diet mixed with barium. The results revealed no abnormalities in the oral phase; however, in the pharyngeal phase, a $10 \%$ residue was observed in the valleculae and a $30 \%$ residue in the pyriform sinus, with a scant amount of aspiration in liquid. Based on VFSS parameters, the patient scored 7 on the penetration aspiration scale (PAS). Unusually, however, the patient

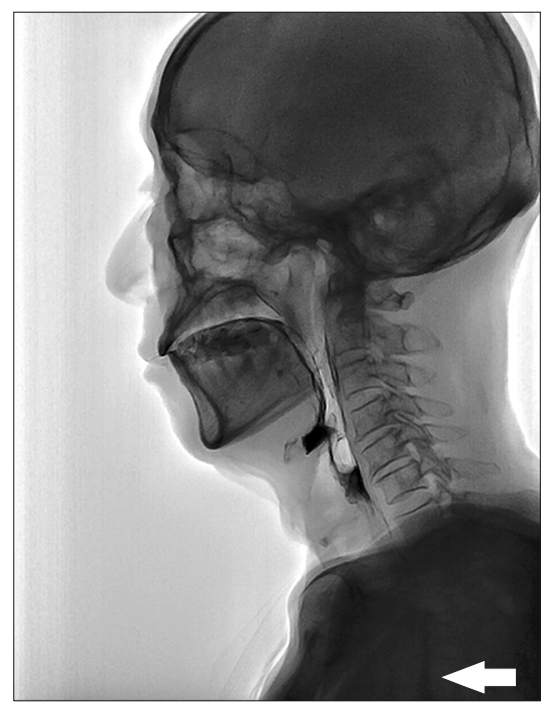

Fig. 1. Captured real-time image of VFSS. The passage of bolus (white arrow) is delayed below the upper esophageal sphincter. pounded on his chest whenever he swallowed the food and complained of "the sensation of food sticking". Descent of the bolus was delayed below the upper esophageal sphincter.(Fig. 1) For detailed verification, VFSS with an anteroposterior view revealed that the esophagus was compressed by a particular structure, causing the lumen to become remarkably narrowed and the bolus to be lodged locally.(Fig. 2) Bolus descended more slowly at the aorta level, with a velocity of about $2.2 \mathrm{~cm} / \mathrm{sec}$ until passing the aorta level. This was about $27 \%$ slower than the normal velocity of $3 \mathrm{~cm} / \mathrm{sec}$ and bolus was lodged for 3 seconds ${ }^{4}$. To search for the presence of esophageal lesions, the gastroenterology department was consulted and esophagogastroduodenoscopy was performed. The results were negative, except for scattered whitish patches at the lower esophagus, which were suspected to be mild esophageal candidiasis. Considering the location and shape of the surrounding structure that compressed the esophagus and caused physiological constriction, the aortic arch was suspected. As such, the cardiology department was consulted to perform chest CT, which revealed no abnormality in thoracic structures other than cardiomegaly, and there was no lesion in the aorta

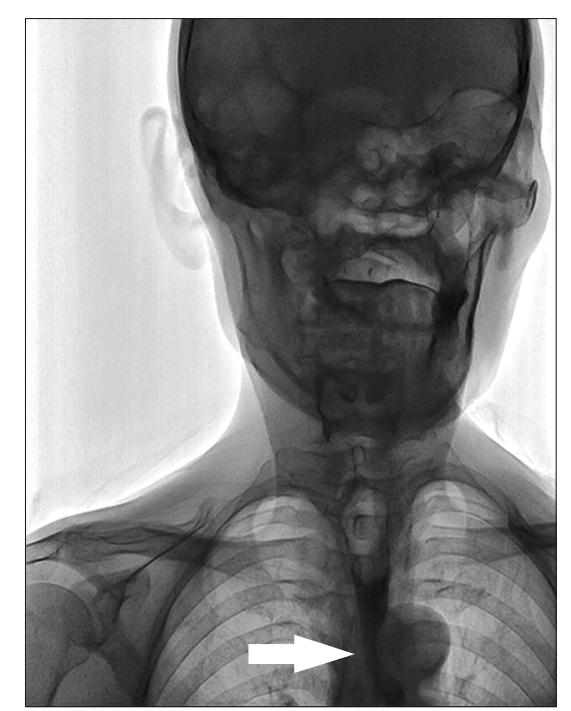

Fig. 2. The esophageal lumen is remarkably compressed (white arrow) by a particular structure. 


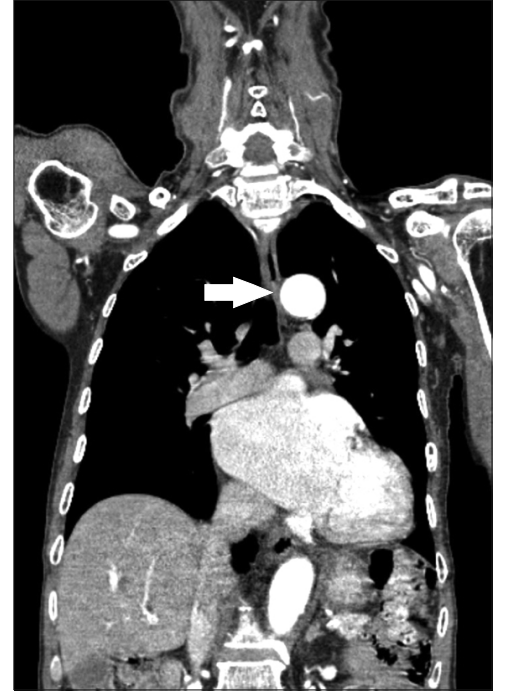

Fig. 3. The aorta is compressing esophagus (white arrow) with the location seen in the VFSS.

itself. However, chest CT showed that the aorta compressed the esophagus at the location observed in the VFSS.(Fig. 3) The cardiology department remarked that there is no particular treatment available for the compression site.

Fifty-two days after the start of swallow therapy, a second VFSS was performed and revealed that valleculae residue was no longer present and residue of the pyriform sinus was reduced to $10 \%$. There was no penetration or aspiration in all types of diet, and the patient scored 1 on the PAS. The patient experienced improved swallowing function but still complained of the sensation of food lodging. The patient did not want further evaluation, so that additional inspection such as manometry was not performed. The authors train patients to perform double-effort swallowing for such symptoms, and decided to observe progression in an outpatient setting.

\section{DISCUSSION}

Dysphagia aortica is a mechanical type of dysphagia caused by external compression of the esophagus by an ectatic, tortuous, or aneurysmal atherosclerotic aorta ${ }^{5}$. However, there were no aortic lesions in this case. Considering that the patient did not experience discomfort before the stroke, it was assumed that the strength of the esophageal muscle, which was normal before the stroke, could have compensated for the pressure caused by physiological constriction by the aorta. There is evidence to suggest that cortical stimulation influences motor function of the upper striated muscle portion of the esophagus ${ }^{6,7}$. Therefore, it is estimated that the patient's brain lesions caused muscle weakness and peristaltic dyscoordination, resulting in the lack of such compensatory action, which led to symptoms and delayed esophageal transition.

There is no single standard diagnostic tool for dysphagia aortica $^{8}$. When dysphagia aortica is suspected, additional diagnostic procedures including radiologic or manometric studies are required ${ }^{2,9}$. Manometry, however, has limitations; it provides only an indirect measure of peristalsis, as the pressure waves recorded do not always correlate with the aboral forces applied to a bolus in the esophagus ${ }^{10}$. Mittal et al. reported that manometry findings do not necessarily correlate with the presence of dysphagia aortica'. Considering the patient's symptoms and image findings, we assumed that neurogenic dysphagia and dysphagia aortica were co-occurring due to stroke.

The treatment of dysphagia aortica varies, depending on the severity of symptoms ${ }^{11}$. Conservative treatments are performed first for mild symptoms of dysphagia because patients are often elderly and have several underlying chronic and limiting diseases ${ }^{2,12}$. In most cases, particularly in the elderly, symptoms may be improved by conservative treatment, such as diet modification (e.g., avoiding food likely to lodge in the esophagus, eating small but frequent meals, and chewing well), and the treatment of underlying heart failure or hypertension?.

However, patients not responsive to conservative treatment or with severe dysphagia symptoms, may respond to surgery, such as transposition of the distal esophagus, separation of the esophagus from the aorta, or repair of the aneurysm ${ }^{8}$. In patients not 
suitable for surgery, percutaneous endoscopic gastrostomy and esophageal dilation with bougies or stents may be attempted ${ }^{13}$. In our case, the sensation of food lodging in the chest persisted; however, the patient refused further evaluation or treatment, and experienced improvement in swallowing without penetration or aspiration and, as such, conservative treatment was continued.

In conclusion, in patients who experience dysphagia after stroke have an unusual sensation of something lodged in their chest, it should be noted that symptoms caused by physiological constriction may overlap in addition to dysphagia caused by stroke.

\section{REFERENCES}

1. Taylor CW, Sinha A, Nightingale JM. Dysphagia and thoracoabdominal aneurysm. Postgrad Med J. 2001;77: 257-8.

2. Wilkinson JM, Euinton HA, Smith LF, Bull MJ, Thorpe JA. Diagnostic dilemmas in dysphagia aortica. Eur J Cardiothorac Surg. 1997;11:222-7.

3. Ponce J, Gaspar E, Del Val A, Garrigues V, Sancho-Tello MJ, Carrasquer J. Dysphagia of vascular origin. Rev Esp
Enferm Dig. 1991;80:53-6.

4. Richter JE, Wu WC, Johns DN, Blackwell JN, Nelson JL, Castell JA, et al. Esophageal manometry in 95 healthy adult volunteers. Dig Dis Sci. 1987;32:583-92.

5. Pape R. Uber einen abnormen verlauf ('tiefe Rechtslage') der mesa aotitischen aorta descendens. Fortschr Roetgenstr. 1932;46:257-69.

6. Aziz Q, Rothwell JC, Barlow J, Thompson DG. Modulation of esophageal responses to magnetic stimulation of the human brain by swallowing and by vagal stimulation. Gastroenterology. 1995;109:1437-45.

7. Diamant NE. A glimpse at the central mechanism for swallowing? Gastroenterology. 1995;109:1700-2.

8. Chan YH, Hung CY, Shieh TY, Wang HY, Chang CW, Shih SC, et al. Nonaneurysmatic dysphagia aortica in the elderly: three case reports and literature review. Int J Gerontol. 2016;10:52-5.

9. Mittal RK, Siskind BN, Hongo M, Flye MW, McCallum RW. Dysphagia aortica: clinical, radiological, and manometric findings. Dig Dis Sci. 1986;31:379-84.

10. Maurer AH. Esophageal transit and gastric emptying. J Nucl Med. 2015;44:1-11.

11. Song SW, Chung JH, Kim SH. A case of dysphagia aortica in an elderly patient. Int J Gerontol. 2012;6:46-8.

12. Hilliard AA, Murali NS, Keller AS. Dysphagia aortica. Ann Intern Med. 2005;142:230-1.

13. Kim JH, Jang SW, Kim DB, Lee HJ, Kim JG, Kwon BJ, et al. A patient with dysphagia due to an aortic aneurysm. Korean Circ J. 2009;39:258-60. 